\title{
Toll-Like receptor 4 promotes the phosphorylation of CRMP2 via the activation of Rho-kinase in MCAO rats
}

\author{
XUE-BO LI ${ }^{1,2}$, MING-XIA DING ${ }^{3}$, CHUN-LI DING ${ }^{2},{\text { LIANG-LIANG } \text { LI }^{2}, \text { JINZHOU FENG }^{4} \text { and XIAO-JUN YU }}^{1}$ \\ ${ }^{1}$ Department of Forensic Medicine, Shantou University Medical College, Shantou, Guangdong 515041; \\ ${ }^{2}$ Key Laboratory of Evidence Identification in Universities of Shandong, Shandong University of Political Science and Law; \\ ${ }^{3}$ Department of Obstetrics and Gynecology, The Second Hospital of Shandong University, Jinan, Shandong 250014; \\ ${ }^{4}$ Department of Neurology, The First Affiliated Hospital of Chongqing Medical University, Chongqing 400016, P.R. China
}

Received July 21,2017; Accepted April 20, 2018

DOI: $10.3892 / \mathrm{mmr} .2018 .8968$

\begin{abstract}
The mechanism associated with Toll-like receptor 4 (TLR4) in neurological injury remains unclear. The aim of the present study was to investigate the pathology of TLR4 in middle cerebral artery occlusion (MCAO)/reperfusion rat models via the regulation of collapsin response mediator protein 2 (CRMP2) phosphorylation. The modified neurological severity score (mNSS) was applied to assess neurological recovery. Immunofluorescence and western blotting were used to detect the protein expressions of TLR4, Rho-associated protein kinase 2 (ROCK-II) and CRMP2 following the intracerebroventricular administration of TLR4-specific agonist, lipopolysaccharide (LPS) and TLR4-neutralizing antibody, the ROCK-II specific inhibitor Y-27632 or LPS+Y-27632 30 min prior to MCAO. The expression levels of TLR4 and the phosphorylation of CRMP2 significantly increased in response to LPS-mediated induction and/or MCAO; however, they were reversed by treatment with LPS+TLR4-neutralizing antibody. Y-27632 decreased the expression of ROCK-II and phosphorylated (p)-CRMP2, and suppressed the increased ROCK-II and p-CRMP2 induced by LPS; however, no effect on the levels of TLR4 expression was observed. The neurological function as measured by mNSS score was reduced in the LPS group when compared with the MCAO group, whereas the LPS+Y-27632 group reversed the reduced neurological function at 7 and 14 days post-MCAO. The results of the present study suggested that TLR4 may promote the phosphorylation of CRMP2 via the activation of ROCK-II in MCAO rats, which further characterizes the pathological mechanism of TLR4 in stroke, and
\end{abstract}

Correspondence to: Dr Xiao-Jun Yu, Department of Forensic Medicine, Shantou University Medical College, 22 Xinling Road, Shantou, Guangdong 515041, P.R. China

E-mail: szfentomology@163.com

Key words: Toll-like receptors 4, Rho-associated protein kinase 2, collapsin response mediator protein 2, middle cerebral artery occlusion/reperfusion, neurological function that modulation of TLR4 could be a potential target to limit secondary post-stroke brain damage.

\section{Introduction}

Stroke is prevalent worldwide, and is among the most frequent causes of adult disability and neurological-associated mortality. Stroke thus affects the quality of life of the patients and their families (1). The majority of strokes (88\%) result from a blockage of blood vessels in the brain, which is known as ischemic stroke; many ischemic strokes occur in the middle cerebral artery (MCA), which has been the focus of previous animal stroke models (2). Thus, the MCA occlusion (MCAO) model results in a cessation of blood flow and subsequent brain infarction in the MCA territory (3); however, the causal mechanisms of stroke require further investigation.

Toll-like receptor 4 (TLR4) expression levels are increased in response to ischemia within MCAO mice models (4). In addition, neurological deficits were significantly decreased in mice deficient in TLR4 when compared with controls (5); however, the mechanism remains unclear. TLRs are widely expressed in innate immune cells, including macrophages and microglia in the central nervous system, which serve a critical role in early innate immunity. Some TLRs, including TLR2, TLR4 and TLR8, are present in microglia, astrocytes, oligodendrocytes and neurons in the brain (6-8). Damaged neuronal structure and deficits in neuroregeneration are common characteristics of post-ischemic injury in stroke. Collapsin response mediator protein 2 (CRMP2) is known to be a neuronal phosphoprotein that regulates microtubule assembly and is involved in anterograde vesicular transport for growth-associated molecules along neuronal microtubules (9). Phosphorylated CRMP2 (p-CRMP2) is unable to bind with microtubules, which in turn inhibits microtubule polymerization and stabilization, thereby inhibiting axonal elongation in neurons (10). Therefore, the phosphorylation of CRMP2 serves a major role in neuroregeneration post-ischemic injury and stroke (11). TLR4 has been demonstrated to be involved in neural stem cell proliferation and serves an important role in neurogenesis (12). Thus, p-CRMP2 may be a candidate for TLR4 regulation. Rho kinase is the enzyme responsible for the phosphorylation of proteins, which participates in microtubule 
assembly and may serve an important role in the TLR4 regulation of p-CRMP2 $(13,14)$; however, the association between TLR4 and p-CRMP2 in stroke conditions of an MCAO ischemic model remains unknown.

In the present study, the mechanism of stroke in a model of TLR4 regulation was investigated. To the best of our knowledge, the present study is the first to report that TLR4 may promote the phosphorylation of CRMP2 within MCAO rat models, and to demonstrate that the effects of phosphorylation may be mediated via the activation of Rho-kinase.

\section{Materials and methods}

Reagents and antibodies. The Rho-associated protein kinase 2 (ROCK-II) inhibitor, Y-27632 (cat. no. 555550; Sigma-Aldrich; Merck KGaA, Darmstadt, Germany), the TLR4 specific agonist lipopolysaccharide (LPS; cat. no. 437628; EMD Millipore, Billerica, MA, USA), CRMP monoclonal antibody (cat. no. ab129082; Abcam, Cambridge, UK) anti-p-CRMP2 polyclonal antibody (cat. no. 9397; Cell Signaling Technology, Inc., Danvers, MA, USA), anti-Rho kinase antibody (cat. no. 9029; Cell Signaling Technology, Inc.), TLR4 monoclonal antibody (cat. no. sc-293072; Santa Cruz Biotechnology, Inc., Dallas, TX, USA), TLR4-neutralizing antibody (cat. no. 16-9917; eBioscience; Thermo Fisher Scientific, Inc., Waltham, MA, USA), and $\beta$-actin (cat. no. sc-47778; Santa Cruz Biotechnology, Inc.) were used.

Animals. A total of 95 adult male Sprague-Dawley rats weighing 240-280 g (10-12 weeks old) were randomly divided into 8 groups: The normal, sham, MCAO/reperfusion, saline, LPS, TLR4-neutralizing antibody, LPS+Y-27632 and Y-27632 groups. The animals were housed in an animal center at a constant temperature of $22 \pm 2^{\circ} \mathrm{C}$, a relative humidity of $50 \pm 10 \%$ and a 12-h light/dark cycle. They were allowed free access to food and water. The present study was approved by the Ethics Committee of the Department of Forensic Medicine, Shantou University (Shantou, Guangdong, China). All experiments were conducted in compliance with the National Institute of Health's Guidelines for the Care and Use of Laboratory Animals (15).

MCAO/reperfusion. The rats in the MCAO/reperfusion, saline, LPS, LPS+TLR4-neutralizing, TLR4-neutralizing antibody ( $n=10$ each group), LPS+Y-27632 and Y-27632 groups $(n=5$ each group) were subjected to MCAO and reperfusion models according to previous studies (16). Briefly, rats were anesthetized with $4 \%$ chloral hydrate intraperitoneally $(300 \mathrm{mg} / \mathrm{kg})$, and MCAO was conducted using an intraluminal filament introduced via the common carotid artery (CCA). A midline surgical incision was made to expose the right CCA, external carotid artery and internal carotid artery (ICA). A 40-mm-long monofilament nylon suture $(\sim 0.26 \mathrm{~mm}$ in diameter $)$ was inserted into the right CCA lumen and gently advanced into the ICA to $\sim 18 \mathrm{~mm}$. The nylon sutures were slowly removed from the artery $2 \mathrm{~h}$ later. The animal's body temperature was continually monitored and maintained at $37^{\circ} \mathrm{C}$ with a homeothermic blanket throughout the surgical procedure. The animals in the sham group $(n=10)$ were treated similarly, with the exception that the filament was not advanced to the origin of the MCA. No treatment performed on the normal group. Neurological function deficits were evaluated following reperfusion when the rats regained consciousness according to the Longa method (17) to evaluate the success of MCAO surgery. Rats without neurological deficits $2 \mathrm{~h}$ following the induction of stroke were excluded from this study.

Intracerebroventricular injection. The intracerebroventricular injection was performed to the saline (control), LPS, LPS+Neutral, Neutral, Y-27632 and LPS+Y-27632 groups ( $n=5$ in each group) according to a previous study (18). The rats were anesthetized and placed in a stereotaxic frame with a mouse head holder. A stainless-steel cannula was implanted into the right lateral cerebral ventricle according to the following coordinates: $1.0 \mathrm{~mm}$ posterior from the Bregma, $1.5 \mathrm{~mm}$ lateral from the midline and at a depth of $4.0 \mathrm{~mm}$ from the skull surface (19). TLR4-neutralizing antibody [200 $\mu \mathrm{g} / \mathrm{kg}$ (20)],LPS [0.2 mg/kg (21)], LPS [0.2 mg/kg]+TLR4-neutralizing antibody [200 $\mu \mathrm{g} / \mathrm{kg}$ ], LPS $(0.2 \mathrm{mg} / \mathrm{kg})+\mathrm{Y}-27632$ [10 mg/kg (22)], and Y-27632 [10 mg/kg] was dissolved in saline to $5 \mu \mathrm{l}$ and injected to each group $30 \mathrm{~min}$ prior to MCAO surgery. The rats in the saline group were injected with saline at the same volume as a control. The injection procedure continued for $10 \mathrm{~min}$, and the microinjector remained for $5 \mathrm{~min}$ prior to withdrawal. The bone wound was closed with bone wax, and then the rats underwent subsequent MCAO surgery. Following $48 \mathrm{~h}$ post-MCAO establishment, the animals were sacrificed, and the brains were removed immediately for further study.

Immunofluorescence. Rats were anesthetized and perfused with PBS and $4 \%$ buffered paraformaldehyde. Following collection of brain tissue, the right cortex was post-fixed in $4 \%$ paraformaldehyde for $24 \mathrm{~h}$ at $4^{\circ} \mathrm{C}$, embedded in paraffin and coronally sectioned at $4 \mu \mathrm{m}$. Sections were permeabilized with $1 \%$ Triton X-100 for $2 \mathrm{~h}$, then blocked with $10 \%$ goat serum (Beyotime Institute of Biotechnology, Shanghai, China) in PBS with $0.3 \%$ Triton $\mathrm{X}-100$ for $30 \mathrm{~min}$ at room temperature. Sections were then incubated with anti-TLR4 (1:2,000), anti-ROCK-II (1:1,000), anti-p-CRMP2 (1:1,000) or saline at $4^{\circ} \mathrm{C}$ overnight. Following washing with PBS, sections were incubated with the secondary antibodies [tetramethylrhodamine- (1:100, Goat anti-Rabbit; cat no. T-2769; Thermo Fisher Scientific, Inc.) or fluorescein isothiocyanate-conjugated (1:500, Goat anti-mouse; cat no. A16079; Thermo Fisher Scientific, Inc.)] for $1 \mathrm{~h}$ at $37^{\circ} \mathrm{C}$. Finally, DAPI was employed for nuclei staining for $10 \mathrm{~min}$ at room temperature. Saline was used as a negative control instead of primary antibody. Images were obtained via fluorescence microscopy (magnification, x200). The Image Pro-Plus 6.0 Software (Media Cybernetics, Inc., Rockville, MD, USA) enabled computer-controlled image acquisition and processing. The relative optical density was calculated, and the relative protein expression was then determined.

Western blotting. Brain tissue was homogenized with lysis buffer (1\% Triton X-100, 1 mM EDTA in 1X PBS, $\mathrm{pH}$ 7.4) containing $200 \mu \mathrm{M}$ phenylmethylsulfonyl fluoride. Homogenates were centrifuged at $15,000 \mathrm{x}$ g for $20 \mathrm{~min}$ at $4^{\circ} \mathrm{C}$, and the supernatants were isolated. Protein concentration was determined using a Bicinchoninic Acid protein 
assay kit. Protein of the samples $(40 \mu \mathrm{g})$ was separated using 10\% SDS-PAGE and transferred to nitrocellulose membranes. The membranes were blocked in 5\% nonfat milk for $1 \mathrm{~h}$ at $37^{\circ} \mathrm{C}$, followed by an overnight incubation at $4^{\circ} \mathrm{C}$ with primary antibodies (anti-TLR4 1:1,000, anti-total CRMP2 1:1,000, anti-p-CRMP2 1:1,500, anti-ROCK2 1:1,000, anti- $\beta$-actin 1:2,000). Following Tris-buffered saline and $0.1 \%$ Tween-20 (TBST) washing and incubation with a secondary antibody (cat. no. 7074, anti-rabbit, 1:5,000; cat. no. 7076 anti-mouse, 1:5,000; Cell Signaling Technology, Inc.) for $1 \mathrm{~h}$ at $37^{\circ} \mathrm{C}$, protein bands were visualized using a chemiluminescence detection kit (cat no. P0018F; Beyotime Institute of Biotechnology, Shanghai, China). $\beta$-actin was used as an endogenous loading control. Relative protein expression levels were reflected by the band density of the target proteins relative to $\beta$-actin. The Quantity One software version 4.4 (Bio-Rad Laboratories, Inc., Hercules, CA, USA) was used to analyze the integrated absorbance value of the band densities.

Modified neurological severity score (mNSS). To further clarify whether Rho-kinase acts on the neurological deficits induced by LPS, neurological function was evaluated using the mNSS at 24 h, 2 and 7 days, and 2 weeks post-MCAO in the MCAO, LPS and LPS+Y-27632 groups ( $\mathrm{n}=5$ each group). The mNSS is a composite of motor, sensory, balance and reflex tests, and is graded on a scale from 0 to 18 (normal score, 0 ; maximal deficit score, 18). A higher score indicates more severe neurological behavioral impairments (23).

Statistical analysis. All experiments were repeated three times. Data were presented as the mean \pm standard deviation. All data were analyzed using one-way analysis of variance followed by Fisher's exact test for post-hoc analyses. $\mathrm{P}<0.05$ was considered to indicate statistically significant difference. Statistical analysis was performed using SPSS 18.0 software (SPSS, Inc., Chicago, IL, USA).

\section{Results}

Expression levels of TLR4 and p-CRMP2 are significantly increased in MCAO rats as observed via western blotting. The results of western blotting demonstrated that the expression levels of TLR4 and p-CRMP2 were significantly increased in the cortex of the MCAO group (TLR4, 0.453 \pm 0.098 ; p-CRMP2, 0.653 \pm 0.078 ) when compared with the normal (TLR4, 0.112 $\pm 0.023 ; \mathrm{P}=0.0037 ; \mathrm{p}-\mathrm{CRMP} 2,0.211 \pm 0.064$; $\mathrm{P}=0.0082)$ and sham groups (TLR4, 0.153 $\pm 0.056 ; \mathrm{P}=0.0074$; p-CRMP2, 0.253 $\pm 0.056 ; P=0.009)$. The TLR4-specific agonist LPS also significantly increased the expression levels of TLR4 $(0.8456 \pm 0.098, \mathrm{P}=0.037)$ and $\mathrm{p}-\mathrm{CRMP} 2$ $(0.948 \pm 0.098, \mathrm{P}=0.024)$ in the cortex when compared with the saline group. The application of the TLR4-neutralizing antibody treatment suppressed the increased expression of TLR4 and p-CRMP2 induced by LPS induction, as observed in the reduction of TLR4 $(0.396 \pm 0.034, \mathrm{P}=0.029)$ and p-CRMP2 $(0.640 \pm 0.054, \mathrm{P}=0.031)$ expression levels in the LPS+TLR4-neutralizing antibody group when compared with the LPS group. When LPS was absent, the expression levels of TLR4 (0.210 $\pm 0.082, \mathrm{P}=0.038)$ and $\mathrm{p}-\mathrm{CRMP} 2(0.352 \pm 0.049$,
$\mathrm{P}=0.017)$ were significantly decreased compared with LPS+ TLR4-neutralizing antibody group (Fig. 1).

Expression levels of TLR4 and p-CRMP2 are significantly increased in MCAO rats as observed by immunofluorescence. The immunofluorescence results revealed that MCAO treatment significantly increased the TLR4 (0.378 \pm 0.054 ; Fig. 2) and p-CRMP2 (0.540 \pm 0.044 , Fig. 3) positive fluorescence in the cortex compared with the normal group (TLR4: $0.162 \pm 0.027, \mathrm{P}=0.075$; p-CRMP2: $0.173 \pm 0.039, \mathrm{P}=0.003$ ). Treatment with LPS also significantly increased the intensity of TLR4 $(0.726 \pm 0.068)$ and p-CRMP2-positive $(0.803 \pm 0.081)$ fluorescence in the cortex when compared with the saline

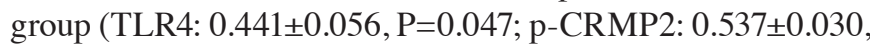
$\mathrm{P}=0.038$ ). Application of the TLR4-neutralizing antibody treatment significantly suppressed the increased TLR4 and p-CRMP2-positive fluorescence induced by LPS induction, as demonstrated by the decrease in TLR4 $(0.604 \pm 0.050$, $\mathrm{P}=0.026)$ and $\mathrm{p}-\mathrm{CRMP} 2$-positive fluorescence $(0.620 \pm 0.093$, $\mathrm{P}=0.008$ ) in the LPS+TLR4-neutralizing antibody group when compared with the LPS group. The expression of TLR4 (0.412 $\pm 0.059, \mathrm{P}=0.007)$ and $\mathrm{p}-\mathrm{CRMP} 2(0.347 \pm 0.038$, $\mathrm{P}=0.005)$ were significantly decreased compared with the LPS+ TLR4-neutralizing antibody group (Figs. 2 and 3).

Rho kinase mediates the role of TLR4 in the phosphorylation of CRMP2. The present study further investigated whether Rho kinase is involved in the increased levels of TLR4-induced CRMP2 phosphorylation. The Rho kinase inhibitor Y-27632 was intracerebroventricularly injected into the MCAO rat models, and the expression of TLR4, ROCK-II and p-CRMP2 were detected via western blotting (Fig. 4). TLR4 protein expression levels exhibited no significant differences between the LPS $(0.457 \pm 0.087)$ and LPS+Y-27632 groups $(0.588 \pm 0.092$, $\mathrm{P}=0.702)$, which suggested that the activation of Rho kinase may not affect the expression of TLR4. However, ROCK-II and p-CRMP2 expression levels were significantly reduced in the

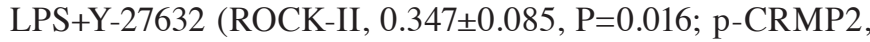
$0.568 \pm 0.054, \mathrm{P}=0.031)$ and $\mathrm{Y}-27632$ (ROCK-II, 0.201 \pm 0.050 , $\mathrm{P}=0.004$; p-CRMP2, $0.333 \pm 0.046, \mathrm{P}=0.022)$ groups when compared with the LPS group (ROCK-II, 0.832 \pm 0.104 ; p-CRMP2, 0.732 \pm 0.098$)$. Notably, Y-27632 treatment alone decreased p-CRMP2, ROCK-II and TLR4 expression levels when compared with the LPS+Y-27632 group ( $\mathrm{P}<0.05$; Fig. 4).

Neurological function is reversed by Rho kinase inhibition. The mNSS score was used to evaluate the neurological function following MCAO surgery. To study the effects of LPS and Rho kinase inhibition on neurological function, LPS or LPS+Y-27632 was injected into rats at several time points including $24 \mathrm{~h}, 2$ and 7 days, and 2 weeks post-MCAO to determine the mNSS score. The results of the present study revealed no significant differences in the mNSS score between the groups $24 \mathrm{~h}$ post-MCAO (MCAO: 11.945 \pm 2.094 ; LPS: $12.997 \pm 1.483$; LPS+Y-27632 12.456 $\pm 2.123, \mathrm{P}=0.866)$. Compared with in the MCAO group, the mNSS score in the LPS group was significantly increased at 2 days $(12.654 \pm 2.006, \mathrm{P}=0.044), 7$ days $(9.567 \pm 1.843, \mathrm{P}=0.039)$ and 14 days $(7.945 \pm 1.046, \mathrm{P}=0.027)$ following MCAO surgery, which means more severe neurological deficits compared with the MCAO group. In addition, Rho kinase inhibition may reverse 

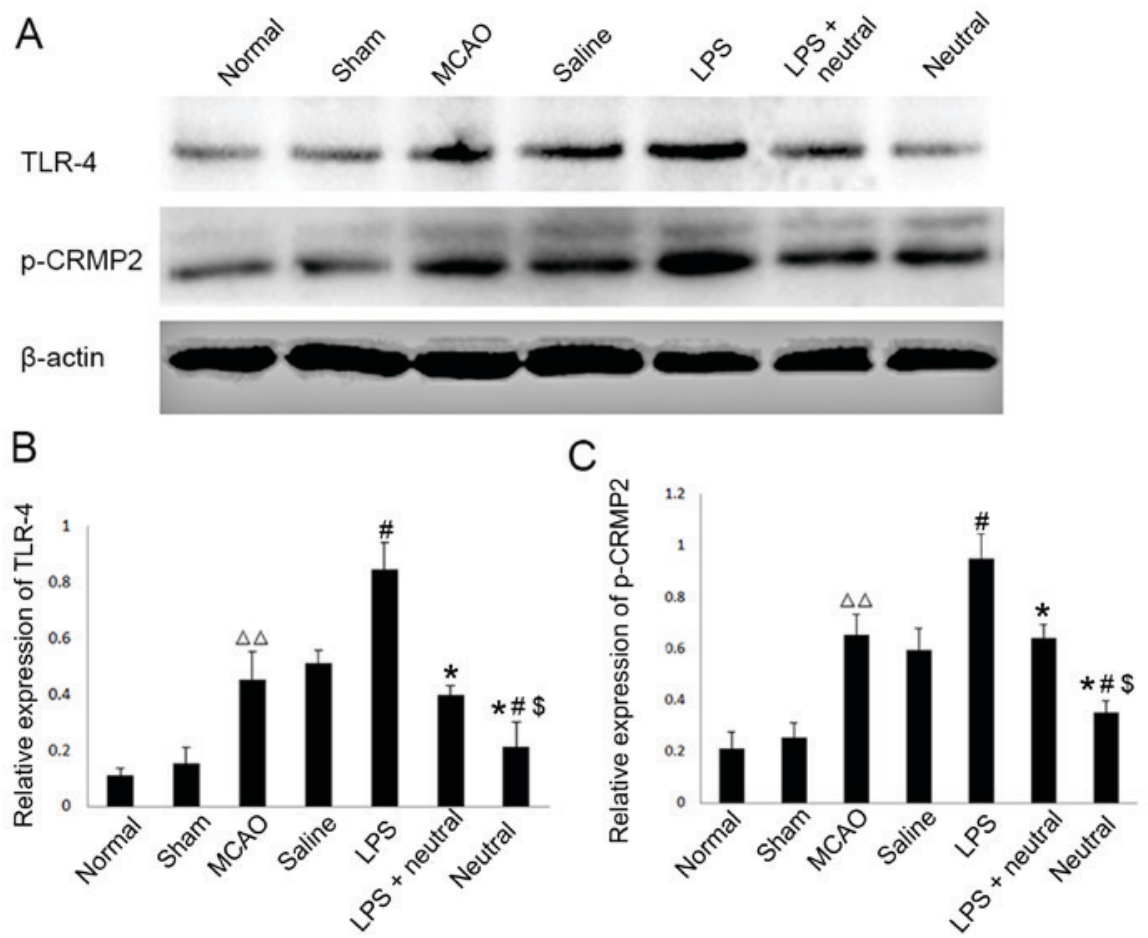

Figure 1. Expression levels of TLR4 and phosphorylation of CRMP2 in the cortex via western blotting. (A) Western blotting was performed to reveal the expression levels of TLR4 and p-CRMP2 in each group. (B) Quantitative analysis of TLR4 expression relative to $\beta$-actin from western blotting as presented in (A). (C) Quantitative analysis of p-CRMP2 expression relative to $\beta$-actin from western blotting a presented in (A). Data are expressed as the mean \pm standard deviation $(n=5) .{ }^{\Delta \Delta} \mathrm{P}<0.01$ vs. the normal group; ${ }^{*} \mathrm{P}<0.05$ vs. the $\mathrm{MCAO}$ group; ${ }^{*} \mathrm{P}<0.05$ vs. the LPS group; ${ }^{\$} \mathrm{P}<0.05$ vs. the LPS+neutral group. LPS, lipopolysaccharide; p-CRMP2, phosphorylated collapsin response mediator protein 2; MCAO, middle cerebral artery occlusion and reperfusion group; Normal, rats that did not undergo MCAO surgery; LPS+neutral, rats treated with LPS and TLR-neutralizing antibody prior to MCAO; Saline, rats administered an intracerebroventricular injection of saline prior to MCAO; Neutral, rats treated with TLR-neutralizing antibody only prior to MCAO; Sham, rats that underwent surgery without MCAO; TLR4, Toll-like receptor 4.
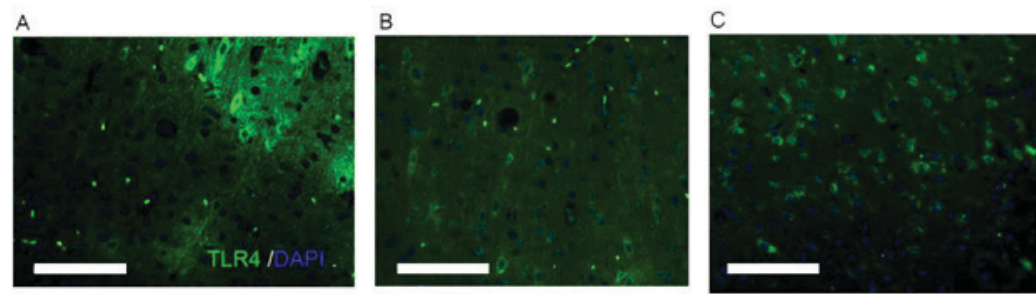

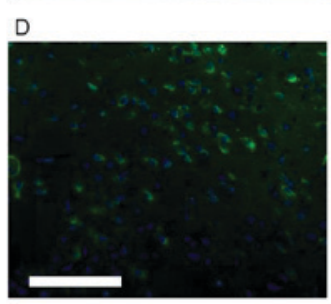

G

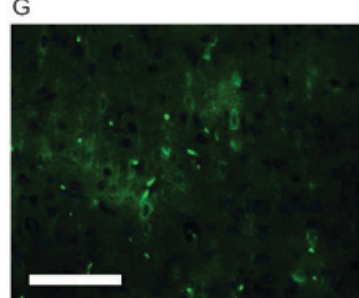

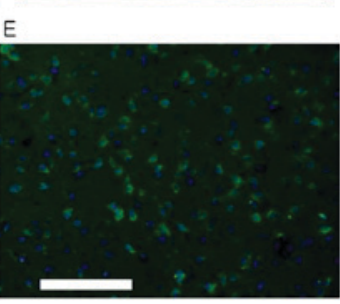

$\mathrm{H}$
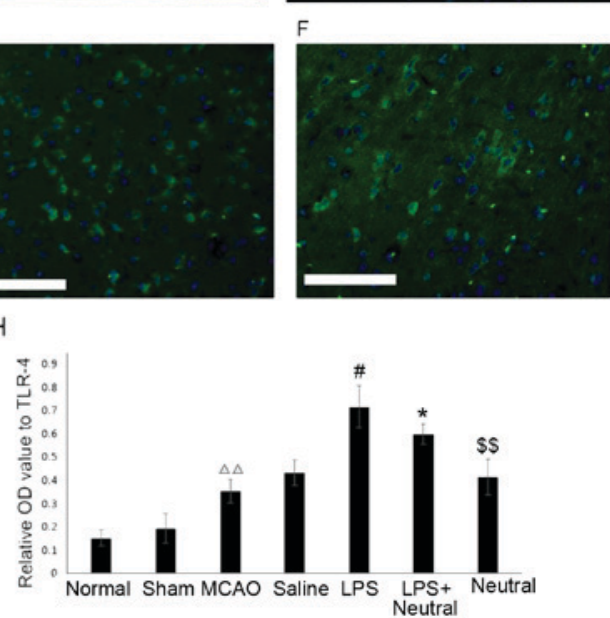

Figure 2. Expression levels of TLR4 in the cortex via immunofluorescence (x200). TLR4-positive expression in the cortex in the (A) Normal, (B) Sham, (C) MCAO, (D) Saline, (E) LPS, (F) LPS+TLR4-neutralizing antibody and (G) TLR4-neutralizing antibody groups. (H) Quantitative analysis of the OD of TLR4-positive cells in the cortex. Data are expressed as the mean \pm standard deviation $(\mathrm{n}=5)$. Scale bar $=100 \mu \mathrm{m} .{ }^{\Delta \Lambda} \mathrm{P}<0.01$ vs. the normal group; ${ }^{\text {\# }} \mathrm{P}<0.05 \mathrm{vs}$. the MCAO group; ${ }^{\prime} \mathrm{P}<0.05$ vs. the LPS group; ${ }^{\$ S} \mathrm{P}<0.01$ vs. the LPS+Neutral group. OD, optical density; LPS, lipopolysaccharide; MCAO, middle cerebral artery occlusion and reperfusion group; Normal, rats that did not undergo MCAO; LPS+Neutral, rats treated with LPS and TLR-neutralizing antibody prior to MCAO; Neutral, rats treated with TLR-neutralizing antibody only prior to MCAO; Sham, rats that underwent surgery without MCAO; Saline, rats administered an intracerebroventricular injection of saline prior to MCAO; TLR4, Toll-like receptor 4. 
A

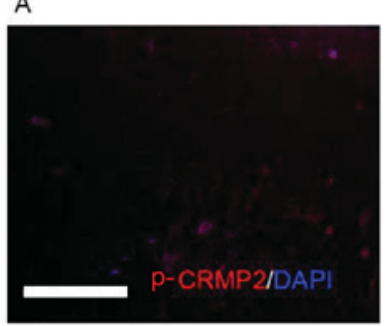

D

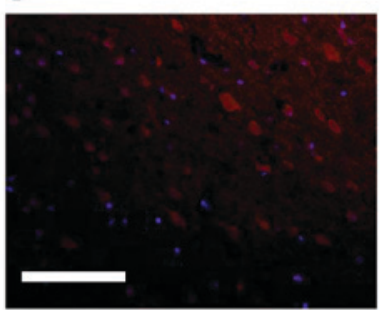

G

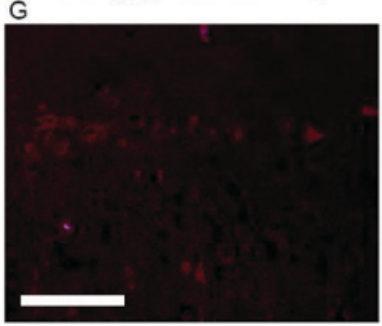

$B$

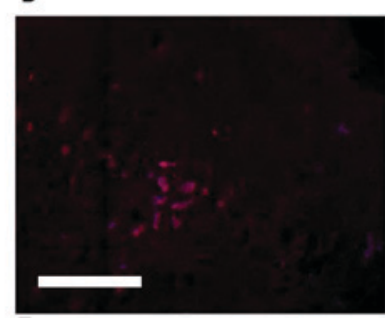

E

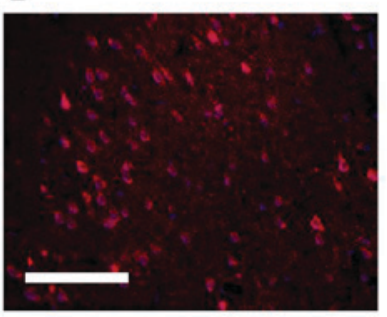

H

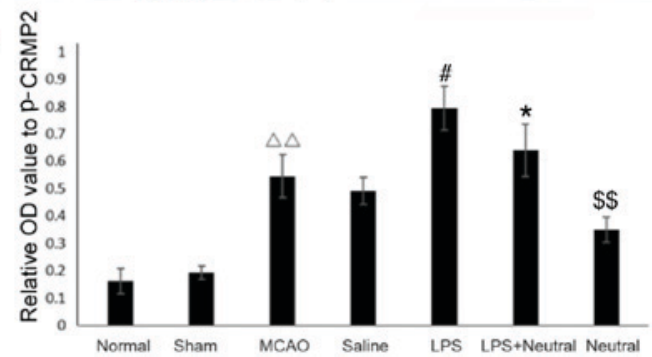

C

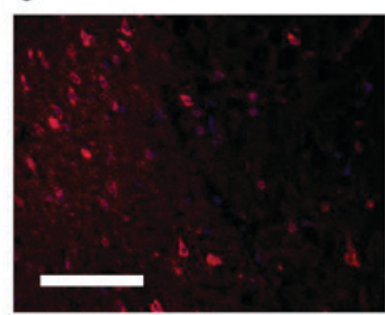

$\mathrm{F}$

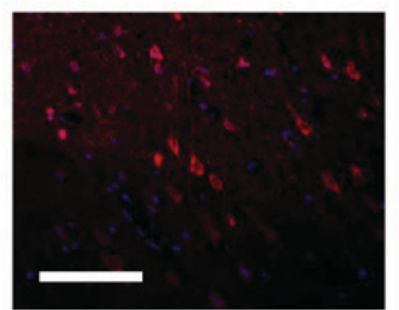

Figure 3. Phosphorylation expression of CRMP2 in the cortex via immunofluorescence (magnification, x200). The p-CRMP2-positive expression in the cortex of the (A) normal, (B) sham, (C) MCAO, (D) saline, (E) LPS, (F) LPS+TLR4-neutralizing antibody and (G) TLR4-neutralizing antibody treatment groups. $(\mathrm{H})$ Quantitative analysis of the OD of TLR4-positive cells in the cortex relative to the OD value. Data are expressed as the mean \pm standard deviation ( $=5$ ). Scale bar $=100 \mu \mathrm{m} .{ }^{\Delta \Delta} \mathrm{P}<0.01$ vs. the normal group; ${ }^{*} \mathrm{P}<0.05$ vs. the MCAO group; ${ }^{*} \mathrm{P}<0.05$ vs. the LPS group; ${ }^{\$ \$} \mathrm{P}<0.01$ vs. the LPS+neutral group. OD, optical density; p-CRMP2, phosphorylated collapsin response mediator protein 2; TLR, Toll-like receptor; LPS, lipopolysaccharide; MCAO, middle cerebral artery occlusion and reperfusion group; Normal, rats that did not undergo MCAO; LPS+ neutral, rats treated with LPS and TLR-neutralizing antibody prior to MCAO; Neutral, rats treated with TLR-neutralizing antibody only prior to MCAO; Saline, rats administered an intracerebroventricular injection of saline prior to MCAO; Sham, rats that underwent surgery without MCAO.

the increased mNSS score exhibited by the LPS group at 7 days $(7.457 \pm 2.075, \mathrm{P}=0.025)$ and 14 days $(4.845 \pm 1.763, \mathrm{P}=0.018)$ post-MCAO, as demonstrated by the significant decrease in the mNSS score observed with the administration of Y-27632 when compared with the LPS group (Fig. 5).

\section{Discussion}

The present study demonstrated that the expression levels of TLR4 and p-CRMP2 were significantly increased in the cortex of rats with MCAO or LPS induction but were suppressed by TLR4-neutralizing antibody treatment or Rho kinase inhibition. Rho kinase specific inhibition reversed the aggravated neurological deficits induced by LPS. The findings of the present study suggested that TLR4 may promote the phosphorylation of CRMP2 via the activation of the Rho-kinase signaling pathway in MCAO, thereby contributing to the pathological mechanism of TLR4 in ischemia and stroke.

TLRs are one of the major components of the innate immune system. Recent studies have demonstrated that TLRs are widely expressed in immune-associated cell types $(24,25)$, which are also located in non-immune cells, including neurons, microglia and epithelial cells (26). TLR expression levels have been reported to be significantly increased in neurons in response to ischemic conditions $(5,7)$. TLR4 inhibition may prevent neuronal death in MCAO rat models (4); however, the mechanism involved has not been clarified. In the present study, the expression levels of TLR4 were significantly increased following MCAO and the TLR4-specific agonist LPS significantly promoted the expression of TLR4, whereas the TLR4-neutralizing antibody reduced the expression levels of TLR4 in MCAO rats. In addition, the results of the present study indicated that neurological function may be inhibited when the expression levels of TLR4 are increased by LPS. These results suggested that TLR4 may serve an important role in the pathogenesis of MCAO.

CRMP2 is a multifunctional adaptor protein that binds cytoskeletal partners to serve a crucial role in microtubule dynamics, neurite outgrowth and retraction, neural differentiation and neurotransmitter release, as well as other important neurophysiological functions (27). CRMP2 phosphorylation by Rho-kinase destabilizes microtubule bundles in the growth cone, in which the cell loses its ability to promote microtubule assembly, ultimately leading to growth cone collapse and the inhibition of neuroregeneration $(28,29)$. Wang et al $(30)$ reported that the expression levels of p-CRMP2 were notably elevated in MCAO rats and induced severe neurological deficits. In the present study, the expression levels of p-CRMP2 in the cortex were significantly increased post-MCAO.

Additionally, the results of the present study demonstrated that the activation of TLR4 by LPS significantly promoted 
A

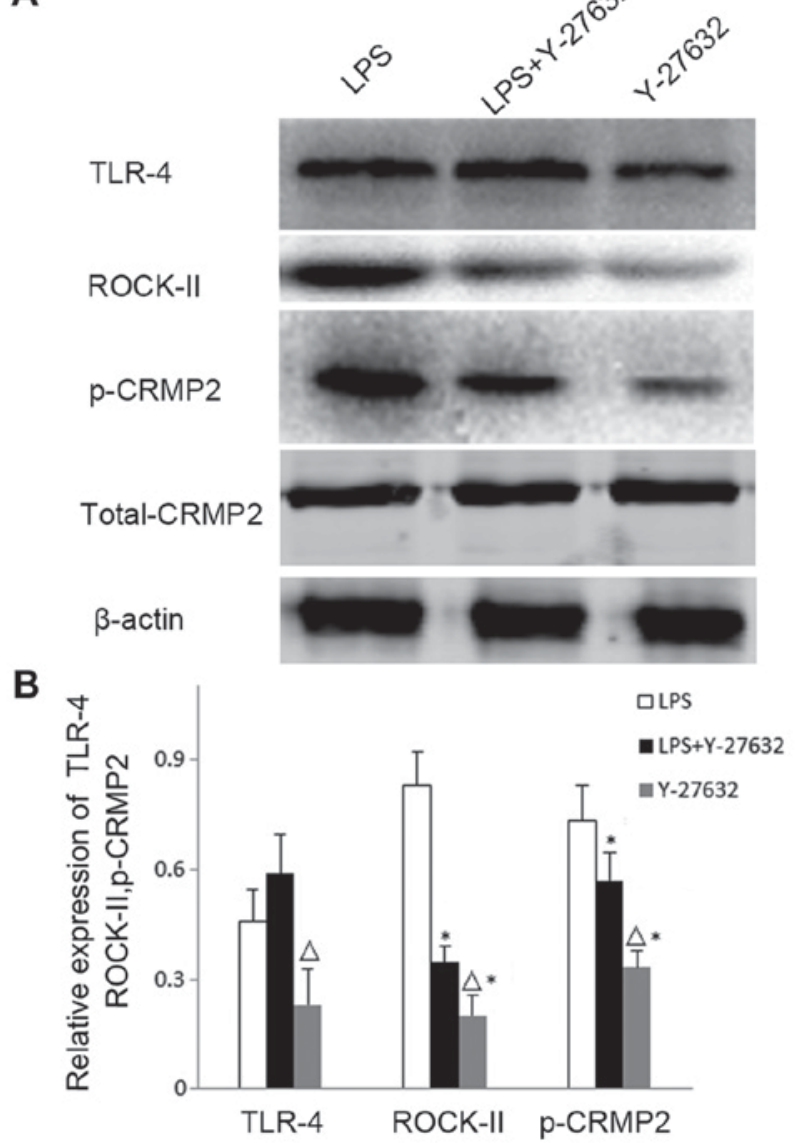

Figure 4. Expression levels of TLR4, ROCK-II and p-CRMP2 in the brain cortex as detected by western blotting. (A) Western blotting was performed to reveal the expression levels of TLR4, the activated Rho-kinase inhibitor ROCK-II and p-CRMP2 in the LPS, LPS+Y-27632 and Y-27632 groups. (B) Quantitative analysis of TLR4, ROCK-II and p-CRMP2 expression levels relative to $\beta$-actin in the cortex from (A). Data are expressed as the mean \pm standard deviation $(\mathrm{n}=5) .{ }^{\Delta} \mathrm{P}<0.05$ vs. the LPS $+\mathrm{Y}-27632$ group; ${ }^{*} \mathrm{P}<0.05$ vs. the LPS group. LPS, lipopolysaccharide; p-CRMP2, phosphorylated collapsin response mediator protein 2; ROCK-II, Rho-associated protein kinase 2; Y-27632, rats treated with the ROCK-II inhibitor Y-27632, LPS+Y-27632, rats treated with LPS and Y-27632; TLR4, Toll-like receptor 4.

the expression levels of p-CRMP2, whereas the inhibition of TLR4 by TLR4-neutralizing antibody significantly reduced the expression of $\mathrm{p}$-CRMP2. These results suggested that TLR4 may regulate the phosphorylation of CRMP2 in MCAO rats. To further investigate the signaling pathway underlying TLR4 regulation associated with the phosphorylation of CRMP2, LPS and the specific Rho-kinase inhibitor, Y-27632, were administered to the brains prior to ischemic injury in the present study. Western blotting revealed that Y-27632 had no effect on the increased expression of TLR4 induced by LPS; however, the expression levels of TLR4, ROCK-II and p-CRMP2 were significantly suppressed by Y-27632 only treatment. These results indicated that the phosphorylation of CRMP2 may be activated by TLR4, which was suppressed following the inhibition of Rho kinase activation. The negative control (normal) was not included in this western blotting experiment, which may present a limitation of the present study.

The results of the present study demonstrated that TLR4 promoted the phosphorylation of CRMP2 via the activation

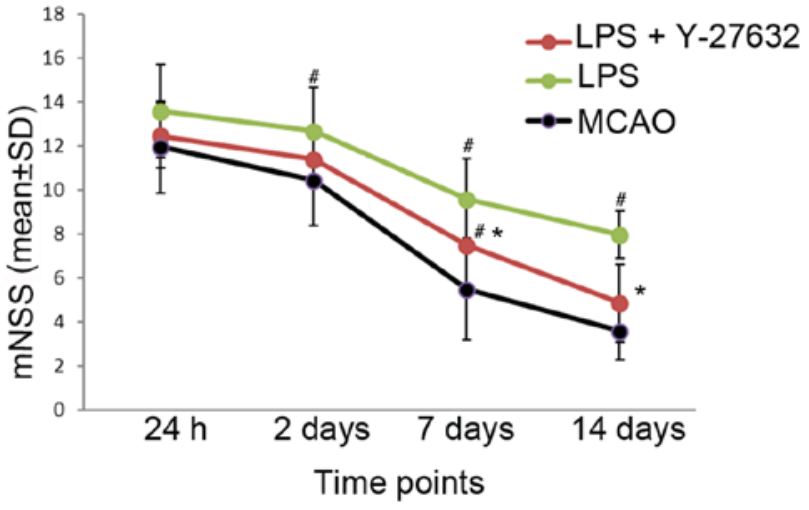

Figure 5. Neurological scores of MCAO rats evaluated using the mNSS scoring method. No significant differences in the mNSS scores were observed at $24 \mathrm{~h}$ post-MCAO between the three groups. The mNSS score in the LPS group was significantly increased at 2, 7 and 14 days post-MCAO when compared with the MCAO group. LPS+Y-27632 treatment reversed the increased mNSS score induced by LPS alone at 7 and 14 days post-MCAO. Data are expressed as the mean \pm standard deviation $(n=5) .{ }^{*} \mathrm{P}<0.05$ vs. the MCAO group at the same time point; ${ }^{*} \mathrm{P}<0.05$ vs. the LPS group. LPS, lipopolysaccharide; Y-27632, the Rho kinase inhibitor; MCAO, middle cerebral artery occlusion and reperfusion; mNSS, modified neurological severity score.

of Rho-kinase. Additionally, the deterioration of neurological deficits associated with LPS intervention may be alleviated by the suppression of Rho-kinase and p-CRMP2. This suggests that the neurological impairments caused by TLR4 may be mediated by Rho-kinase and p-CRMP2.

However, additional experiments are required to support the conclusions of the present study. For example, further investigation of the direct interaction between p-CRMP and Rho-kinase, except for intervention with specific inhibitors, is required. In addition, it is also essential that histopathological analysis, such as Evans Blue/hematoxylin and eosin staining is conducted to study the levels of apoptosis/necrosis in neuronal cells and further evaluate the brain damage, which may support the results of behavioral neurological testing conducted in the present study.

In conclusion, the present study demonstrated that TLR4 may promote the phosphorylation of CRMP2 in MCAO rats, which may have been mediated via the activation of Rho-kinase. This helps to further clarify the pathogenesis of TLR in stroke; modulation of TLR4 could be a potential target to limit secondary post-stroke brain damage in future clinical applications.

\section{Acknowledgements}

Not applicable.

\section{Funding}

The present study was supported by the Department of Education, Guangdong Government under the Top-tier University Development Scheme for Research and Control of Infectious Diseases (grant no. 2015064), National Natural Science Foundation Council of China (grant nos. 81072508 and 81501634, Natural Science Foundation of Shandong Province (grant no. ZR2014HQ018), Project of Shandong Province Higher Educational Science and Technology Program (no. J17KA240), China Postdoctoral Science Foundation (no. 2017M612701) and The Special Project of 
Technical Innovation about Social and People's Livelihood in Chongqing (no. cstc2015shmszx0017).

\section{Availability of data and materials}

The datasets used and/or analyzed during the current study are available from the corresponding author on reasonable request.

\section{Authors' contributions}

XY and XL conceived the idea of the study and designed research; LL and JF analysed the data; CD and XL interpreted the results; MD wrote the paper, raised the animals and performed the western blot protocol; all authors performed research, discussed the results and revised the manuscript.

\section{Ethics approval and consent to participate}

The present study and experimental protocol was established, according to the ethical guidelines of the Helsinki Declaration and was approved by the Ethics Committee of Department of Forensic Medicine, Shantou University (Shantou, China).

\section{Consent for publication}

Not applicable.

\section{Competing interests}

The authors declare that they have no competing interests.

\section{References}

1. Mensah GA, Norrving B and Feigin VL: The global burden of stroke. Neuroepidemiology 45: 143-145, 2015.

2. Herson PS and Traystman RJ: Animal models of stroke: Translational potential at present and in 2050. Future Neurol 9: 541-551, 2014.

3. Sierra C, Coca A and Schiffrin EL: Vascular mechanisms in the pathogenesis of stroke. Curr Hypertens Rep 13: 200-207, 2011.

4. Xu X, Wen Z, Zhao N, Xu X, Wang F, Gao J, Jiang Y and Liu X: MicroRNA-1906, a Novel regulator of toll-like receptor 4 ameliorates ischemic injury after experimental stroke in mice. J Neurosci 37: 10498-10515, 2017.

5. Tang SC, Arumugam TV, Xu X, Cheng A, Mughal MR, Jo DG, Lathia JD, Siler DA, Chigurupati S, Ouyang X, et al: Pivotal role for neuronal Toll-like receptors in ischemic brain injury and functional deficits. Proc Natl Acad Sci USA 104: 13798-13803, 2007.

6. Tang SC, Yeh SJ,Li YI, Wang YC, Baik SH, Santro T, Widiapradja A, Manzanero S, Sobey CG, Jo DG, et al: Evidence for a detrimental role of TLR8 in ischemic stroke. Exp Neurol 250: 341-347, 2013.

7. Olson JK and Miller SD: Microglia initiate central nervous system innate and adaptive immune responses through multiple TLRs. J Immunol 173: 3916-3924, 2004.

8. Lehnardt S, Lehmann S, Kaul D, Tschimmel K, Hoffmann O, Cho S, Krueger C, Nitsch R, Meisel A and Weber JR: Toll-like receptor 2 mediates CNS injury in focal cerebral ischemia. J Neuroimmunol 190: 28-33, 2007.

9. Fukata Y,Itoh TJ,Kimura T, Ménager C, Nishimura T, Shiromizu T, Watanabe H, Inagaki N, Iwamatsu A, Hotani H and Kaibuchi K CRMP-2 binds to tubulin heterodimers to promote microtubule assembly. Nat Cell Biol 4: 583-591, 2002.

10. Yoshimura T, Kawano Y, Arimura N, Kawabata S, Kikuchi A and Kaibuchi K: GSK-3beta regulates phosphorylation of CRMP-2 and neuronal polarity. Cell 120: 137-149, 2005

11. Gim SA, Sung JH, Shah FA, Kim MO and Koh PO: Ferulic acid regulates the AKT/GSK-3 $\beta /$ CRMP-2 signaling pathway in a middle cerebral artery occlusion animal model. Lab Anim Res 29: 63-69, 2013.
12. Ye $\mathrm{Y}, \mathrm{Xu} \mathrm{H}$, Zhang $\mathrm{X}, \mathrm{Li} \mathrm{Z}, \mathrm{Jia} \mathrm{Y}, \mathrm{He} \mathrm{X}$ and Huang JH Association between toll-like receptor 4 expression and neural stem cell proliferation in the hippocampus following traumatic brain injury in mice. Int J Mol Sci 15: 12651-12664, 2014.

13. Julian L and Olson MF: Rho-associated coiled-coil containing kinases (ROCK): Structure, regulation, and functions. Small GTPases 5: e29846, 2014.

14. Yoneda A, Multhaupt HA and Couchman JR: The rho kinases I and II regulate different aspects of myosin II activity. J Cell Biol 170: 443-453, 2005.

15. National Research Council: Guide for the care and use of laboratory animals. 8th edition. National Academy Press, Washington, DC, 2011. https://grants.nih.gov/grants/olaw/guide-for-the-careand-use-of-laboratory-animals.pdf.

16. Arumugam TV, Salter JW, Chidlow JH, Ballantyne CM, Kevil CG and Granger DN: Contributions of LFA-1 and Mac-1 to brain injury and microvascular dysfunction induced by transient middle cerebral artery occlusion. Am J Physiol Heart Circ Physiol 287: H2555-H2560, 2004.

17. Longa EZ, Weinstein PR, Carlson S and Cummins R: Reversible middle cerebral artery occlusion without craniectomy in rats. Stroke 20: 84-91, 1989.

18. Xiong X, Barreto GE, Xu L, Ouyang YB, Xie X and Giffard RG: Increased brain injury and worsened neurological outcome in interleukin- 4 knockout mice after transient focal cerebral ischemia. Stroke 42: 2026-2032, 2011.

19. Iwasa T, Matsuzaki T, Tungalagsuvd A, Munkhzaya M, Kawami T, Kato T, Kuwahara A, Yasui T and Irahara M: Effects of ovariectomy on the inflammatory responses of female rats to the central injection of lipopolysaccharide. J Neuroimmunol 277: 50-56, 2014.

20. Ma Y, Zhang X, Bao H, Mi S, Cai W, Yan H, Wang Q, Wang Z, Yan J, Fan GC, et al: Toll-like receptor (TLR) 2 and TLR4 differentially regulate doxorubicin induced cardiomyopathy in mice. PLoS One 7: e40763, 2012.

21. Gong G, Bai S, Wu W, Hu L, Liu Y, Niu J, Dai X, Yin L and Wang X: Lrg participates in lipopolysaccharide preconditioning-induced brain ischemia injury via TLR4 signaling pathway. J Mol Neurosci 54: 20-26, 2014

22. Rikitake Y, Kim HH, Huang Z, Seto M, Yano K, Asano T, Moskowitz MA and Liao JK: Inhibition of rho kinase (ROCK) leads to increased cerebral blood flow and stroke protection. Stroke 36: 2251-2257, 2005.

23. Sugawara T, Ayer R, Jadhav V and Zhang JH: A new grading system evaluating bleeding scale in filament perforation subarachnoid hemorrhage rat model. J Neurosci Methods 167: 327-334, 2008

24. Kawai T and Akira S: The role of pattern-recognition receptors in innate immunity: Update on toll-like receptors. Nat Immunol 11: 373-384, 2010.

25. Medzhitov R and Janeway CA Jr: Innate immunity: The virtues of a nonclonal system of recognition. Cell 91: 295-298, 1997.

26. Doi Y, Mizuno T, Maki Y, Jin S, Mizoguchi H, Ikeyama M, Doi M, Michikawa M, Takeuchi H and Suzumura A: Microglia activated with the toll-like receptor 9 ligand $\mathrm{CpG}$ attenuate oligomeric amyloid \{beta\} neurotoxicity in in vitro and in vivo models of Alzheimer's disease. Am J Pathol 175: 2121-2132, 2009.

27. Hensley K, Venkova K, Christov A, Gunning W and Park J: Collapsin response mediator protein-2: An emerging pathologic feature and therapeutic target for neurodisease indications. Mol Neurobiol 43: 180-191, 2011.

28. Brown M, Jacobs T, Eickholt B, Ferrari G, Teo M, Monfries C, Qi RZ, Leung T, Lim L and Hall C: Alpha2-chimaerin, cyclin-dependent Kinase 5/p35, and its target collapsin response mediator protein-2 are essential components in semaphorin 3A-induced growth-cone collapse. J Neurosci 24: 8994-9004, 2004.

29. Arimura N, Ménager C, Kawano Y, Yoshimura T, Kawabata S, Hattori A, Fukata Y, Amano M, Goshima Y, Inagaki M, et al: Phosphorylation by rho kinase regulates CRMP-2 activity in growth cones. Mol Cell Biol 25: 9973-9984, 2005.

30. Wang T, Wu X, Yin C, Klebe D, Zhang JH and Qin X: CRMP-2 is involved in axon growth inhibition induced by RGMa in vitro and in vivo. Mol Neurobiol 47: 903-913, 2013.

This work is licensed under a Creative Commons Attribution-NonCommercial-NoDerivatives 4.0 International (CC BY-NC-ND 4.0) License. 